\title{
The Involvement of RCAS1 in Creating a Suppressive Tumor Microenvironment in Patients with Salivary Gland Adenocarcinoma
}

\author{
Magdalena Dutsch-Wicherek • Agata Lazar • \\ Romana Tomaszewska
}

Received: 8 June 2010 / Accepted: 5 August 2010 / Published online: 20 August 2010

(C) The Author(s) 2010. This article is published with open access at Springerlink.com

\begin{abstract}
The tumor microenvironment is the tissue that determines the growth and progression of the tumor as well as its ability to initiate metastases. The aim of the present study has been to evaluate the role of RCAS1 in creating the suppressive tumor microenvironment in cases of parotid adenocarcinoma. The tissue samples of salivary gland adenocarcinomas and their stroma and the palatine tonsils which constituted the reference tissue sample group were obtained during routine surgical procedures. The immunoreactivity of RCAS1, CD3, CD25, CD68, CD69, and Foxp 3 antigens was then evaluated by using the immunohistochemistry method. The patient's consent was obtained in each case. A statistically significantly higher RCAS1 immunoreactivity level was found in the adenocarcinoma tissue samples in comparison to that found in the stromal tissue samples. A statistically significantly higher RCAS1 immunoreactivity was also identified in the adenocarcinoma tissue samples derived from patients who had lymph node metastases in comparison to patients without such metastases. Additionally, we observed the presence of RCAS1-positive macrophages in the stromal tissue samples. The infiltration of CD68-positive cells was significantly stronger in the adenocarcinoma and stromal tissue slides than in the reference group tissue slides; moreover, the infiltration was a good deal more prominent in the stromal tissue than in the adenocarcinoma tissue. The CD68
\end{abstract}

\footnotetext{
M. Dutsch-Wicherek ( $₫)$

Otolaryngology Head and Neck Surgery Department, Jagiellonian University,

Kraków, Poland

e-mail: mowicher@gmail.com
}

A. Lazar $\cdot$ R. Tomaszewska

Pathology Department, Jagiellonian University,

Kraków, Poland immunoreactivity levels in both the tumor and stromal tissue samples were found to be significantly higher in those patients who had lymph node metastases than in the patients without such metastases. Additionally, the infiltration of CD3- and CD25-positive cells was more prominent in the reference tissue slides than in the adenocarcinoma and stromal tissue slides, and was stronger in the adenocarcinoma tissue than in the stromal tissue. Furthermore, the infiltration of Foxp3-positive cells was seen exclusively in the stroma whereas it was not even detected in the adenocarcinoma tissue. Lastly, the Foxp3-positive cell infiltration was more prominent in the stromal tissue than in the reference group tissue. The present study demonstrates that RCAS1 expression by both tumor cells and tumorassociated macrophages may participate in creating the immunosuppressive microenvironment in parotid gland adenocarcinoma, thus promoting tumor development as well as metastases.

Keywords Salivary gland adenocarcinoma .

Tumor microenvironment $\cdot$ RCAS1-positive macrophages

\section{Introduction}

The tumor microenvironment makes up the stroma of the neoplasm and is the tissue that determines the growth and progression of the tumor as well as its ability to initiate metastases [1]. It has been established that the tumor microenvironment inhibits the infiltration and activity of $\mathrm{T}$ lymphocytes and creates local immunosuppression [2]; however, precisely which component of the tumor microenvironment is responsible for tumor immunopathogeneity, remains unknown [3]. The tumor microenvironment consists of many distinct types of cells, including endothelial 
cells and their precursors, pericytes, smooth muscle cells, fibroblasts, carcinoma-associated fibroblasts, myofibroblasts, neutrophils, eosinophils, basophils, mast cells, T and B lymphocytes, natural killer cells, and antigenpresenting cells (APC) such as macrophages and dendritic cells [4]. The creation of an immunosuppressive microenvironment by the tumor enables the escape of tumor cells from the host immunological surveillance and blocks any effective anticancer immune response. Moreover, the range of the tumor immunosuppressive activity is not just local but general. The process is realized by many factors, such as FasL and HLA-G; it is also realized either through recruiting tumor-associated macrophages and Treg cells or by the expression of yet other factors such as RCAS1. RCAS1 is expressed by various cancer cells. The major function of RCAS1 expression is to inhibit activated immune cells such as T and B lymphocytes and NK cells and the induction of the apoptosis of these cells. RCAS1 is therefore responsible for tumor escape from the host immunological surveillance and for the creation of immune tolerance for tumor cells $[5,6]$. It has also been postulated that RCAS1 is involved in the remodeling of the tumor microenvironment in cases of uterine cervical cancer. Certainly, Sonoda et al. have demonstrated that RCAS1 expression is significantly related to VEGF expression and microvessel density and through the regulation of angiogenesis may participate in tissue remodeling [7]. In patients with ovarian cancer, the level of RCAS1 expression was inversely related to the presence of the vimentin-positive cells in the stromal tissue, and it has therefore been suggested that RCAS1 is involved in the remodeling of the tumor microenvironment [7-9]. RCAS1 expression has also been identified in the healthy portion of the microenvironment of head and neck squamous cell carcinoma and it has been a predictor of disease recurrence [10].

As both Treg cells and tumor-associated macrophages seem to be involved in creating the suppressive tumor microenvironment, the aim of our study has been to determine the presence of these cells in salivary gland adenocarcinomas and their stromas. We also focused on the potential role of RCAS1 expression in the tumor microenvironment of patients with salivary adenocarcinoma relative to the presence of immune cells and their activity.

\section{Materials and Methods}

The Group of Patients

\section{Patients with Salivary Adenocarcinoma}

All the tissue samples were derived from patients from whom salivary gland tumors were removed during surgery performed in the Department of Otolaryngology of the Jagiellonian University. The patient's consent was obtained in each case. Additionally, approval for the research program was granted from the Ethical Committee of the Jagiellonian University in Krakow: KBET/90/B/2005. We recruited 35 patients to our study from those who had undergone the surgery between January 2000 and December 2007. All the tissue samples were histopathologically verified. Pathological analysis using the classical hematoxylin and eosin staining techniques after fixation in a formalin of the surgically removed material was performed in the Pathology Department of the Jagiellonian University by an experienced pathologist. The clinical characteristics of the subjects are presented in Table 1.

\section{Reference Group}

We chose to use the palatine tonsils removed from patients with recurrent tonsillitis as a reference tissue group. From these samples we evaluated the epithelium lining of the tonsils alone and not of the lymphoid tissue.

\section{Immunohistochemical Analysis}

Immunohistochemical analysis was performed in the Pathology Department of the Jagiellonian University. Five-micrometer slides from each case were stained to visualize the expression of RCAS1, CD3-, CD25-, CD68-, CD69, and Foxp3-positive cells. In all cases, immunohistochemistry was performed by applying the Envision method using Dako Autostainer. The following antibodies were applied: mouse monoclonal antibody Anti- RCAS1 (Medical and Biological Laboratories, Naka-ku Nagoya, Japan in DAKO Antibody Diluent with Background Reducing Components-DAKO, Denmark, dilution 1:1000), monoclonal mouse antibody ImmunOTM (MP Biomedicals, Inc., clone 1A12 in dilution 1:1000), CD68 (DAKO, clone PG-M1 in dilution 1:50), CD3 (Novocastra, clone IF6 in dilution: 1:50), CD25 (Interleukin-2 Receptor, NCL-CD25-305, Novocastra in dilution 1:25), and Foxp3, according to the manufacturer's instructions. Visualization of the reaction products was performed using AEC (3amino-9-ethyl-carbazole) as a chromogen (AEC Substrate Chromogen ready-to-use, DAKO, Denmark) for $10 \mathrm{~min}$ at room temperature. Sections were then counterstained with hematoxylin and mounted in glycergel. As a positive control, a tonsil specimen was taken for RCAS1. All stainings were performed with the same procedure except that the primary antibody as a negative control was omitted. RCAS1 expression was evaluated in entire slides in the area of the tumor and in the stroma, as follows: 0 - no reactivity; $+1-$ weak, when observed any (also granular in paranuclear region) cytoplasmic staining pattern (in up to $10 \%$ of positive 
Table 1 The clinical characteristics of the patients with salivary adenocarcinomas

\begin{tabular}{|c|c|}
\hline The number of patients & 35 \\
\hline$M^{\mathrm{a}}$ & 14 \\
\hline $\mathrm{W}^{\mathrm{a}}$ & 21 \\
\hline Age range (average) & $12-80(55.9)$ \\
\hline $\mathrm{M}^{\mathrm{a}}$ & $31-80(56.9)$ \\
\hline $\mathrm{W}^{\mathrm{a}}$ & $12-79(55.2)$ \\
\hline \multicolumn{2}{|l|}{ Tumor stage $^{\mathrm{b}}$} \\
\hline I-II & 29 \\
\hline III-IV & 6 \\
\hline \multicolumn{2}{|c|}{ The presence of lymph node metastases } \\
\hline No & 29 \\
\hline $\mathrm{N}+$ & 6 \\
\hline \multicolumn{2}{|l|}{ Tumor size } \\
\hline $\mathrm{T} 1-\mathrm{T} 2$ & 32 \\
\hline $\mathrm{T} 3-\mathrm{T} 4$ & 3 \\
\hline \multicolumn{2}{|l|}{ Tumor grade } \\
\hline G1 & 2 \\
\hline $\mathrm{G} 2$ & 24 \\
\hline G3 & 9 \\
\hline \multicolumn{2}{|l|}{ Localization of the tumor } \\
\hline Parotid gland & 30 \\
\hline Nasal cavity & 2 \\
\hline Oral cavity & 2 \\
\hline Orbit & 1 \\
\hline \multicolumn{2}{|l|}{ Histological types of tumors } \\
\hline Adenocarcinoma & 13 \\
\hline Adenocarcinoma acinocellulare & 16 \\
\hline Adenocarcinoma apocrinale & 2 \\
\hline Adenocarcinoma mucinosum & 1 \\
\hline Adenocarcinoma tubulare & 1 \\
\hline Adenocarcinoma basaloides & 1 \\
\hline Adenocarcinoma gelatinosum & 1 \\
\hline \multicolumn{2}{|l|}{ Tumors } \\
\hline Primary & 30 \\
\hline Recurrence & 5 \\
\hline
\end{tabular}

${ }^{\mathrm{a}} M$ men; $W$ women

${ }^{\mathrm{b}}$ Tumor stage was evaluated according to TNM classification 6th edition, 2002

cells); +2-marked cytoplasmic (sometimes together with membranous) staining in $11-30 \%$ of the cells); +3 - high expression (more than $30 \%$ of positive cells). Variable scales were used to evaluate the amount of each type of cell semiquantitatively, depending on their overall number in the specimen- an average cell number per $1 \mathrm{hpf}$ (high power field, objective magnification $\mathrm{x} 40$ ). The average number of $\mathrm{CD} 25+$, $\mathrm{CD} 3+, \mathrm{CD} 68+$, and $\mathrm{CD} 69+$ cells were thus estimated as follows: 0 - lack of positive cells; +1 - single positive cells in the specimen; $+2-2-5$ positive cells per $1 \mathrm{hpf} ;+3$ - more than 5 positive cells $/ 1 \mathrm{hpf}$.
We were able to distinguish the macrophages from the stromal cells by their respective morphologies, that is, their characteristic size, nuclear-cytoplasmic ratio, and cell nucleus picture.

Statistical Analysis The distribution of variables in the study groups of patients checked with the use of the Shapiro-Wilk test showed that each of the patients was in fact different from normal. The statistical significance between the groups was determined by the Kruskal-Wallis test, one-way analysis of variance by ranks. The Mann-Whitney $U$ test was then used as applicable. All statistical analyses were carried out with the Statistica 8.0 software program. A $p$ value $<0.05$ was considered indicative of statistical significance.

\section{Results}

The Immunoreactivity of Antigens in Adenocarcinomas and Their Stroma

The Analysis of CD3 and CD25 Immunoreactivity in the Tissue Slides Examined

CD3 antigen immunoreactivity was found in $77.2 \%$ of the adenocarcinoma samples and in $8 \%$ of the stromal samples (Fig. 1a). CD25 antigen immunoreactivity was demonstrated in $31.4 \%$ of the adenocarcinoma tissue slides and in $2.8 \%$ of the stromal slides; it was also observed in salivary gland secretions (Fig. 1b).

The Analysis of CD68 Immunoreactivity in the Tissue Slides Examined

CD68 antigen immunoreactivity was demonstrated in 20\% of the adenocarcinoma tissue slides and in $30 \%$ of the stromal tissue slides (Fig. 2).

The Analysis of CD69 Immunoreactivity in the Tissue Slides Examined

CD69 antigen immunoreactivity was demonstrated in $2.8 \%$ of the adenocarcinoma tissue slides and in $5 \%$ of the stromal tissue slides (Fig. 3).

\section{The Analysis of RCAS1 Immunoreactivity in the Tissue} Samples Examined

RCAS1 antigen immunoreactivity was identified in $65.7 \%$ of the adenocarcinoma tissue slides and in $14.2 \%$ of the stromal tissue slides (Fig. 4).

We also discovered that the macrophages infiltrating the stroma were expressing RCAS1 (Fig. 5). 
Fig. 1 The immunoreactivity of CD3 and CD25 antigens in adenocarcinoma and stromal tissue slides
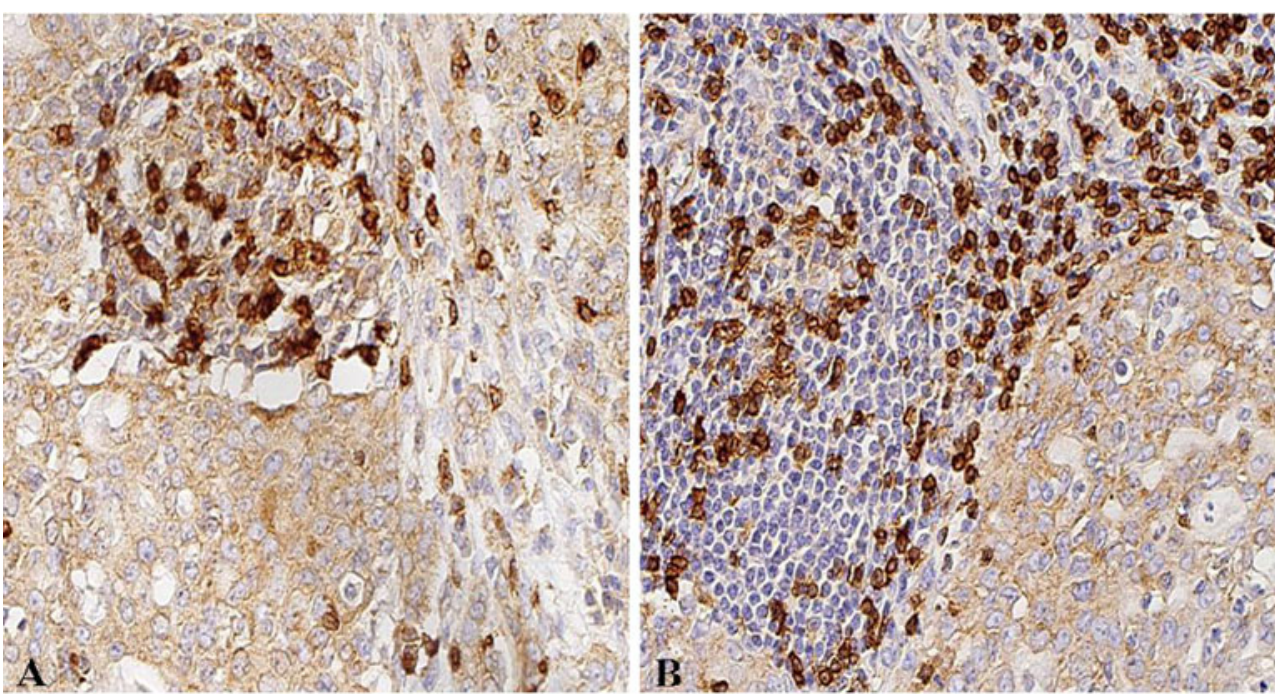

The Comparison of the Immunoreactivity Levels of the Antigens Analyzed in Adenocarcinoma and Their Stromas

The results obtained regarding the immunoreactivity of the antigens analyzed in the tissue samples of adenocarcinomas and their stromas are presented in Table 2.

A statistically significantly higher number of CD3- and CD25-positive cells were identified in the adenocarcinoma tissue slides than in the stromal slides. In turn, a statistically significantly higher number of CD68-positive cells were identified in the tumor microenvironment than in the tumor tissue slides. The immunoreactivity of the Foxp3 antigen was not identified in the adenocarcinoma tissue, but was observed exclusively in the lymphocytes of the stromal tissue.

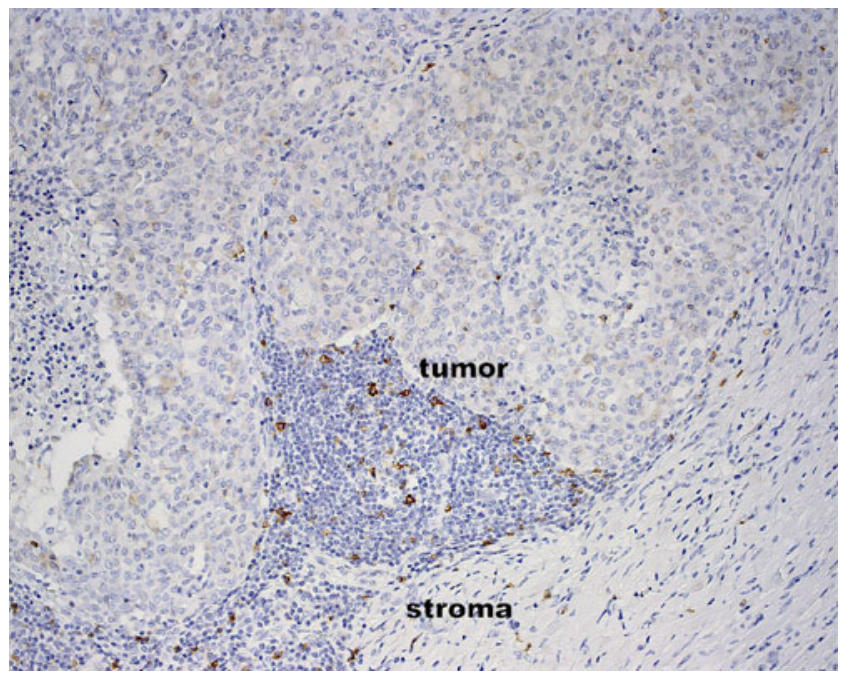

Fig. 2 The immunoreactivity of CD68 in adenocarcinoma and stromal tissue slides
The Comparison of Antigen Immunoreactivity in the Adenocarcinoma and Reference Group Tissue Slides

The results obtained are presented in Table 3.

The Comparison of the Levels of Antigen Immunoreactivity in the Stroma and Reference Group Tissues

The results obtained are presented in Table 4.

The analysis of antigen immunoreactivity with respect to tumor grade and size did not reveal any significant differences.

The analysis of the levels of antigen immunoreactivity in patients with regional lymph node metastases revealed significant differences. The results obtained are presented in Table 5 .

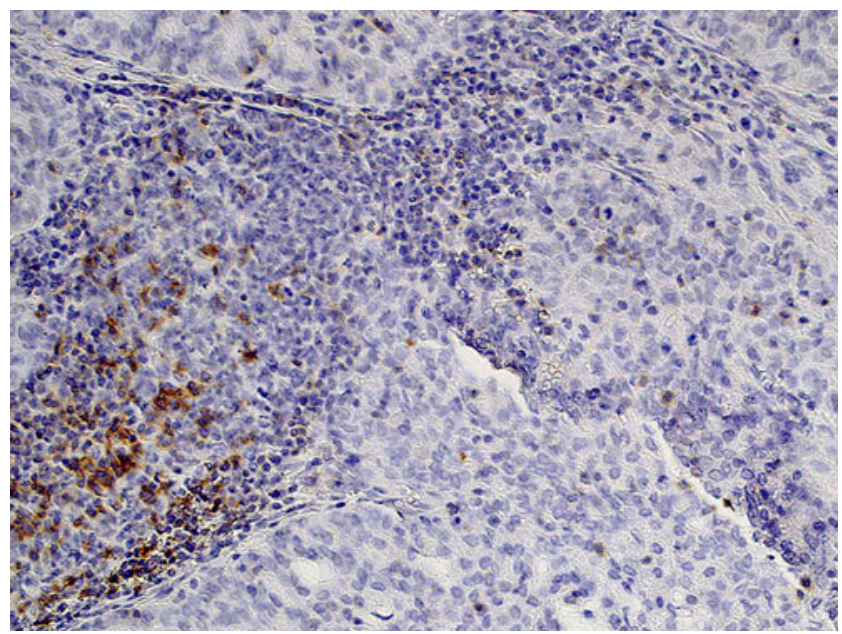

Fig. 3 The immunoreactivity of CD69 antigen immunoreactivity in adenocarcinoma and stromal tissue slides 
Fig. 4 RCAS1 immunoreactivity in adenocarcinoma and stromal tissue slides
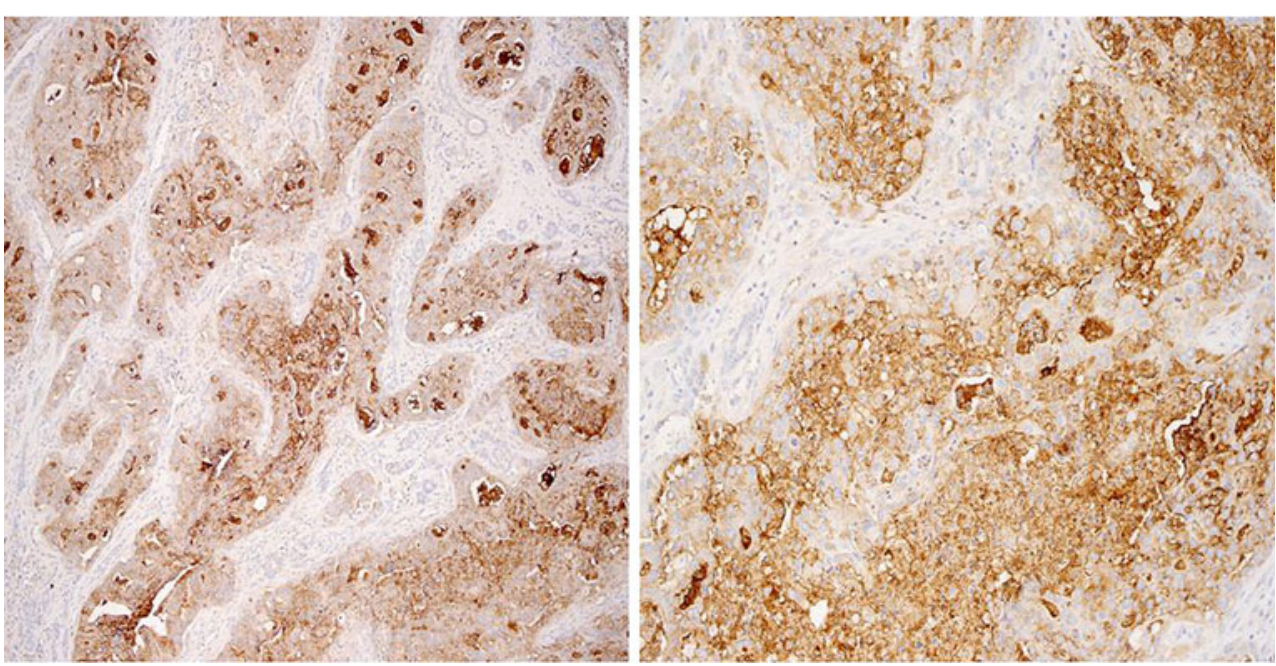

\section{Discussion}

In the present study statistically significantly higher levels of RCAS1 immunoreactivity were identified in the adenocarcinoma tissue samples derived from patients who had lymph node metastases in comparison to the levels found in the tissues from patients without such metastases. To our knowledge this is the first investigation concerning RCAS1 expression in salivary gland tumors and their stromas. Our observation coincides with what has been presented in other reports on the expression of RCAS1 in patients with head and neck squamous cell carcinoma, where RCAS1 expression was significantly higher in patients with the presence of lymph node metastases and was identified as higher in cases where the extra-capsular spread of infiltrated lymph nodes was observed $[10,11]$. The relation between RCAS1 expression and the presence of lymph node metastases was observed in many malignant neoplasms (including esophageal, gastric, gallbladder, pancreatic, colon and uterine cervix)

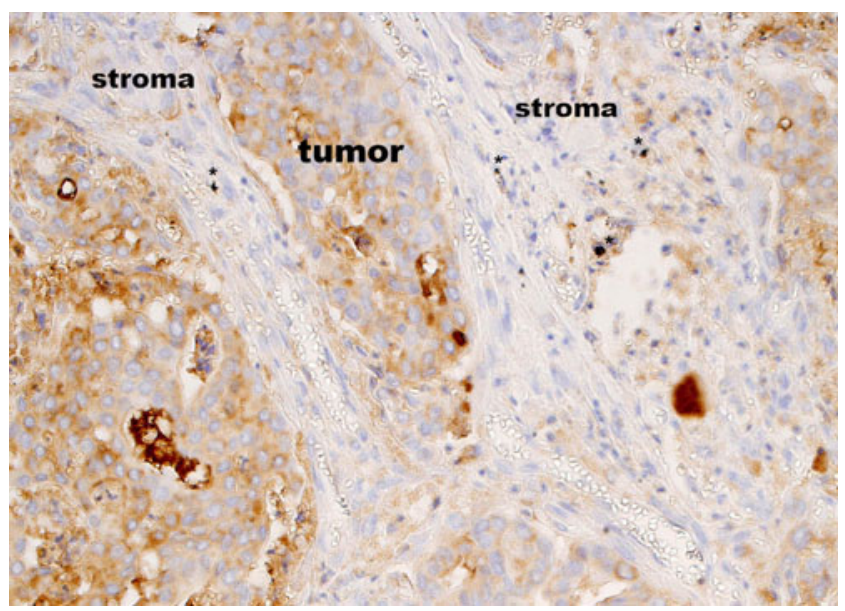

Fig. 5 RCAS1 -positive macrophages in adenocarcinoma and in its stroma
[7, 12-17]. The major function of RCAS1 expression is to inhibit activated immune cells such as $\mathrm{T}$ and B lymphocytes and NK cells and to induce their apoptosis; RCAS1 would therefore be responsible for tumor escape from the host immunological surveillance and the creation of immune tolerance for tumor cells $[5,6]$. It has also been postulated that RCAS1 maybe involved in tumor microenvironment remodeling in cases of uterine cervix cancer $[7,9]$. In cases of cervical cancer, RCAS1 expression has been associated with VEGF expression and microvessel density in a statistically significant way; it has therefore been proposed that RCAS1 maybe involved in the control of angiogenesis [7]. In the present study we also observed the level of mmunoreactivity of RCAS1 in the stroma of adenocarcinoma and it was statistically significantly lower than the level of RCAS1 immunoreactivity observed in the cancer tissue samples. RCAS1 immunoreactivity in the stromal samples was significantly higher in patients who had lymph node metastases in comparison to those patients without such metastases. This observation coincides with the observations presented in our previous report on the stroma of squamous cell carcinoma of the head and neck. RCAS1 has been detected not only in cancer cells, but also

Table 2 The levels of CD3, CD25, CD68, CD69, Foxp3, RCAS1 antigen immunoreactivity in the adenocarcinoma and stromal tissue slides

\begin{tabular}{llll}
\hline Antigen & $\begin{array}{l}\text { Adenocarcinoma } \\
\text { Median (IQR) }\end{array}$ & $\begin{array}{l}\text { Stroma Median } \\
(\mathrm{IQR})\end{array}$ & $p$ value \\
\hline $\mathrm{CD} 3$ & $1.37(2.0)$ & $0.25(0)$ & $p<0.001$ \\
$\mathrm{CD} 25$ & $0.42(0)$ & $0.05(0)$ & $p<0.001$ \\
CD68 & $0.57(0)$ & $0.74(0)$ & 0.01 \\
CD69 & $0.11(0)$ & $0.08(0)$ & $\mathrm{NS}$ \\
Foxp3 & $0(0)$ & $0.22(0)$ & $\mathrm{NS}$ \\
RCAS1 & $1.62(2)$ & $0.4(0)$ & $p<0.001$ \\
\hline
\end{tabular}

IQR Interquarter Range; NS Non-statistically Significant 
Table 3 The comparison of the levels of antigen immunoreactivity in adenocarcinoma and the tissue reference group. Table 3 presents the results of the analysis of the immunoreactivity of the antigens in the adenocarcinoma and reference tissue slides. The results obtained were non-parametric and so the Mann-Whitney's test was used for the statistical analysis; $p<0.05$ was accepted as statistically significant. The results are presented as median and interquarter range (IQR) values. The immunoreactivity of the antigens was evaluated according to the scale presented in the Methods section

\begin{tabular}{llcl}
\hline Antigen & $\begin{array}{l}\text { Adenocarcinoma } \\
\text { Median (IQR) }\end{array}$ & $\begin{array}{l}\text { Reference group } \\
\text { Median (IQR) }\end{array}$ & $p$ value \\
\hline CD3 & $1.3(2)$ & $1.5(2)$ & 0.0008 \\
CD25 & $0.42(0)$ & $2.4(3)$ & $p<0.001$ \\
CD68 & $0.57(0)$ & $0.1(0)$ & $p<0.001$ \\
CD69 & $0.11(0)$ & $0(0)$ & $p<0.001$ \\
Foxp3 & $0(0)$ & $0(0)$ & $p<0.001$ \\
RCAS1 & $1.62(2)$ & $2.4(3)$ & NS* \\
\hline
\end{tabular}

$I Q R$ Interquarter Range; NS Non-statistically Significant

in the healthy portion of the tumor microenvironment, such as the stroma and the epithelium adjacent to the tumor. Moreover, RCAS1 stromal expression has been associated with a higher risk of local recurrence of the disease [10].

There are two activities involving RCAS1 expression that seem to be important in tumor development. One activity is related to its participation in maintaining indolent chronic inflammation in the tumor and its healthy stroma which promotes tumor growth [18]. The second activity of RCAS1 helps to create immune tolerance against tumor antigens, including the elimination of the proper immune anticancer response, by inhibiting the activated $\mathrm{T}$ and $\mathrm{B}$ lymphocytes and NK cells and inducing their apoptosis [5]. Additionally, the immunoregulatory role of RCAS1 does not just affect the tumor and its microenvironment, but can

Table 4 The comparison of the levels of antigen immunoreactivity in the stromal and reference tissue samples. Table 4 presents the results of the analysis of the immunoreactivity of the antigens in the stroma and reference tissue slides. The results obtained were non-parametric and so the Mann-Whitney's test was used for the statistical analysis; $p<0.05$ was accepted as statistically significant. The results are presented as median and interquarter range (IQR) values. The immunoreactivity of the antigens was evaluated according to the scale presented in the Methods section

\begin{tabular}{llll}
\hline Antigen & $\begin{array}{l}\text { Stroma Median } \\
(\text { IQR) }\end{array}$ & $\begin{array}{l}\text { Reference group } \\
\text { Median (IQR) }\end{array}$ & $p$ value \\
\hline CD3 & $0.25(0)$ & $1.5(2)$ & $p<0.001$ \\
CD25 & $0.05(0)$ & $2.4(3)$ & $p<0.001$ \\
CD68 & $0.74(0)$ & $0.1(0)$ & 0.01 \\
CD69 & $0.08(0)$ & $0(0)$ & 0.04 \\
Foxp3 & $0.22(0)$ & $0(0)$ & NS \\
RCAS1 & $0.4(0)$ & $2.4(3)$ & $p<0.001$ \\
\hline
\end{tabular}

$I Q R$ Interquarter Range; NS Non-statistically Significant
Table 5 Analysis of the immunoreactivity of CD3, CD25, CD68, CD69, Foxp3, and RCAS1 antigens in the adenocarcinoma and stromal tissue slides in patients with regional lymph node metastases $(\mathrm{N}+$ group) and in patients without metastases (N0 group)

\begin{tabular}{llll}
\hline Antigen & $\begin{array}{l}\mathrm{N} 0(n=29) \\
\text { Median (IQR) }\end{array}$ & $\begin{array}{l}\mathrm{N}+(n=6) \\
\text { Median (IQR) }\end{array}$ & $p$ value \\
\hline CD3 Tumor & $1.0(1.0)$ & $1.0(1.0)$ & $\mathrm{NS}$ \\
CD3 Stroma & $0(1)$ & $0(1)$ & $\mathrm{NS}$ \\
CD25 Tumor & $0(1)$ & $0(1)$ & $\mathrm{NS}$ \\
CD25 Stroma & $0(1)$ & $0(1)$ & $\mathrm{NS}$ \\
CD68 Tumor & $0(1)$ & $1(1)$ & 0.03 \\
CD68 Stroma & $0(1)$ & $2(1)$ & 0.02 \\
CD69 Tumor & $0(0)$ & $0(0)$ & $\mathrm{NS}$ \\
CD69 Stroma & $0(0)$ & $0(0)$ & $\mathrm{NS}$ \\
Foxp3 Tumor & $0(0)$ & $0(0)$ & $\mathrm{NS}$ \\
Foxp3 Stroma & $0(0)$ & $0(0)$ & $\mathrm{NS}$ \\
RCAS1 tumor & $1(1)$ & $2(1)$ & $p<0.001$ \\
RCAS1 Stroma & $0(0)$ & $0(0)$ & 0.03 \\
\hline
\end{tabular}

$I Q R$ Interquarter Range; NS Non-statistically Significant

have systemic consequences as well. RCAS1 can be secreted in a soluble form, sRCAS1, by ectodomain shedding and has been identified in the blood sera of patients with head and neck squamous cell carcinoma, adenocarcinoma, and benign tumors such as mixed tumor [19-21]. The level of sRCAS1 was statistically significantly higher in patients with malignant tumors than in those with benign tumors [19, 20, 22, 23]. A higher sRCAS1 blood serum level has been identified in patients who had lymph node metastases than in those without such metastases, as well as in patients with malignant gastroesophageal tract tumors, uterine cervix tumors, head and neck squamous cell carcinomas, and salivary gland adenocarcinomas [20, 24]. Furthermore, the sRCAS1 blood serum level was seen to decrease in patients following radical surgery and to increase in patients suffering from cancer relapse [20].

In the present study we observed that the infiltration of CD3- and CD25-positive cells was more prominent in the reference group tissue sample slides than in the adenocarcinoma and stromal tissue slides; it was also found to be stronger in adenocarcinoma than in the stromal slides.

IL-2R $\alpha(C D 25)$ is a basic receptor for the proliferation of T lymphocytes and its decreased expression may induce immune suppression [25]. TIL (tumor infiltrating lymphocytes) are present in most solid tumors and their microenvironments and most commonly consist of CD3-positive T lymphocytes. The specific TIL-mediated tumor cytolysis has been demonstrated in cases of breast cancer, indicating that IL-2 maybe the trigger for this response [26]. Such a cytolysis has also been observed in patients with malignant 
melanoma and kidney cancer and was enhanced by IL-2 stimulation. IL-2 activates T lymphocytes after the interaction with IL-2 (IL-2R) on the surface of T lymphocytes [27, 28]. In the microenvironment of breast cancer TIL lymphocytes were activated (expressed CD69, CD43, and CD38), but were typified by significantly decreased IL-2R expression (decreased expression of CD25 antigen). Additionally, the production and secretion of IL-2 was decreased or absent in the breast cancer microenvironment [29]. Thus, the decreased expression of CD25 as well as IL-2 may result from the induction of the immune tolerance in the tumor microenvironment [30]. In sum, we observed the presence of TIL with the immunoreactivity of $\mathrm{CD} 3$, in both the adenocarcinoma and stromal tissue samples. These cells were activated (CD69 antigen immunoreactivity was significantly higher in the adenocarcinoma and stromal tissue than in the reference group tissue) but their cytotoxic function was impaired as indicated by decreased CD25 antigen immunoreactivity. This observation seems to confirm the presence of selectively inhibited lymphocytes within the neoplasm and its microenvironment. Such a profile of CD69- and CD25-antigen expression has been observed by Sheu et al. and has been identified as a phenotype of selectively suppressed lymphocytes infiltrating the tumor and its microenvironment [31].

The immune tolerance to the tumor antigens is created by proteins and cells. Tumor- associated macrophages significantly outnumber the antigen-presenting cells in solid tumors [32-35]. It has further been demonstrated that tumor-associated macrophages may promote tumor growth and metastases [32-35]. In our study we observed a significantly higher number of CD68-positive cells (macrophages) in both the adenocarcinoma and the stromal tissue samples than in the tissue samples of the control group. Furthermore, the number of CD68-positive cells in adenocarcinoma and stromal tissue was higher in those patients who had lymph node metastases than in the patients without such metastases. The positive correlation between tumor-associated macrophages and poor prognosis has been demonstrated in cases of many types of cancer, and these macrophages were present from the early stages of tumor development $[33,35,36]$. The tumor and its environment express chemotactic agents for macrophages [37]; macrophages in turn secrete growth factors and angiogenic factors which are able to modify the tumor microenvironment and affect tumor growth $[35,38,39]$. Additionally, in our study the macrophages were found to exhibit RCAS1 immunoreactivity. RCAS1-expressing macrophages have also been observed in the bone marrow where they play a pivotal role in the regulation of the process of erythropoiesis by eliminating improper cells by inducing their apoptosis [39, 40]. It has been demonstrated that macrophages stimulated with LPS (lipopolysacharide) enhance RCAS1 expression and induce apoptosis of the progenitor cells of erythroblast line through
RCAS1 [39]. It has further been shown that RCAS1 can only be identified on activated monocytes [40]. Interestingly, RCAS1 expression has been detected on macrophages in various types of inflammatory liver diseases. The type of macrophage found in the inflamed tissue depends on the particular stage of inflammation. At the beginning stages of inflammation, macrophages act as antigen-presenting cells. At more advanced stages, macrophages start to express different types of antigens, such as RCAS1, and their function becomes immunosuppressive [41]. In addition, the presence of RCAS1-positive macrophages has been demonstrated in the microenvironment of nasal polyps with extensive inflammation [42, 43]. Because RCAS1-positive macrophages represent a cell population able to negatively regulate the activity of immune system cells, their presence in the tumor microenvironment may also contribute to creating local immunosuppression in both the tumor and its microenvironment by inhibiting activated lymphocytes that possess RCAS1R (namely, T and B lymphocytes and NK cells).

The immune tolerance to tumor growth is also realized by the recruitment of $\mathrm{T}$ regulatory (Treg) cells to the tumor and its microenvironment. For instance, Treg cells are able to suppress proliferating effector $\mathrm{T}$ lymphocytes $[44,45]$. In order to explore the tumor microenvironment and to be able to localize both the antigens expressed by the cells and the cells themselves, we performed immunohistochemistry. This method, however, did not enable us to evaluate the type of cell precisely (we could assess the immunoreactivity of only one antigen for the tissue slide); it was therefore not possible to identify Treg cells within the tumor and its microenvironment. We have, however, observed Foxp3-expressing lymphocytes exclusively in tumor stroma, and the number of these cells was significantly higher in the stroma than in the reference group tissue. If the cells represented Treg cells it would indicate enhanced Treg cell infiltration within the tumor microenvironment. These cells would then be responsible for creating the local immunosuppressive microenvironment in the stroma that inhibits the response of T effector cells. Lastly, it was shown that these cells interact with macrophages as IL-10 triggers the expression of $\mathrm{B} 7-\mathrm{H} 4$ antigen and the B7-H4-positive macrophages exert an immunosuppressive effect similar to that of Treg cells [2].

In conclusion, we have demonstrated in this study that RCAS1 expression by tumor cells and tumor-associated macrophages within the tumor microenvironment when coupled with a both a decreased number of CD3-positive cells and the presence of Foxp3-positive lymphocytes, may participate in creating the immunosuppressive microenvironment in salivary gland adenocarcinomas.

Acknowledgments This work was supported by the State Committee for Scientific Research (KBN), Grant Number N403 032 31/2079, and also in part by the Jagiellonian University, Grant Number WL/ZKL/26/L. 
Open Access This article is distributed under the terms of the Creative Commons Attribution Noncommercial License which permits any noncommercial use, distribution, and reproduction in any medium, provided the original author(s) and source are credited.

\section{References}

1. Witz IP (2009) The tumor microenvironment: the making of a paradigm. Cancer Microenvironment 2:S9-S17

2. Kryczek I, Zou L, Rodriguez P, Zhu G, Wei S, Mottram P, Brumlik M, Cheng P, Curie T, Myers L, Lackner A, Alvarez X, Ochoa A, Chen L, Zou W (2007) B7-H4 expression identifies a novel suppressive macrophage population in human ovarian carcinoma. JEM 203:871-881

3. Zou W (2005) Immunosuppressive networks in the tumor microenvironment and their therapeutic relevance. Nat Rev Cancer 5:263-274

4. Coussens LM, Werb Z (2002) Inflammation and cancer. Nature 420:860-867

5. Sonoda K, Nakashima M, Kaku T, Kamura T, Nakano H, Watanabe T (1996) A novel tumor-associated antigen expressed in human uterine and ovarian carcinomas. Cancer 77:1501-1509

6. Sonoda K, Miyamoto S, Hirakawa T, Yagi H, Yotsumoto F, Nakashima M, Watanabe T, Nakano H (2006) Clinical significance of RCAS1 as a biomarker of uterine cancer. Gynecol Oncol 103:924-931

7. Sonoda K, Miyamoto S, Hirakawa T, Yagi H, Yotsumoto F, Nakashima M, Watanabe T, Nakano H (2005) Invasive potency related to RCAS1 expression in uterine cervical cancer. Gynecol Oncol 99:189-198

8. Sonoda K, Miyamoto S, Kobayashi H, Ogawa S, Okugawa K, Taniguchi S, Wake N (2009) The level of RCAS1 expression is inversely correlated with the number of vimentin-positive stromal cells in epithelial ovarian cancer. Int J Gynecol Cancer 19:838-843

9. Kokkinos MI, Wafai R, Wong MK, Newgreen DF, Thompson EW, Waltham M (2007) Vimentin and epithelial-mesenchymal transition in human breast cancer - observations in vitro and in vivo. Cells Tissues Organs 185:191-203

10. Dutsch-Wicherek M, Tomaszewska R, Lazar A, Wicherek $Ł$, Skladzień J (2009) The association between RCAS1 expression in laryngeal and pharyngeal cancer and its healthy stroma with cancer relapse. BMC Cancer 9:35

11. Dutsch-Wicherek M (2010) RCAS1, MT, and vimentin as potential markers of tumor microenvironment remodeling. Am J Reprod Immunol 63:181-188

12. Tsujitani S, Saito H, Oka S, Sakamoto T, Kanaji S, Tatebe S, Ikeguchi M (2002) Prognostic significance of RCAS1 expression in relation to the infiltration of dendritic cells and lymphocytes in patients with esophageal carcinoma. Dig Dis Sci 52:549-554

13. Fukuda K, Tsujitani S, Maeta Y, Yamaguchi K, Ikeguchi M, Kaibara N (2002) The expression of RCAS1 and tumor infiltrating lymphocytes in patients with T3 gastric carcinoma. Gastric Cancer 5:220-227

14. Nakamura Y, Yamazaki K, Oizumi S, Nakashima M, Watanabe T, Dosaka-Akita H, Nishimura M (2004) Expression of RCAS1 in human gastric carcinoma: a potential mechanism of immune escape. Cancer Sci 95:260-265

15. Oshikiri T, Hida Y, Miyamoto M, Hashida H, Katoh K, Suzuoki M, Nakakubo Y, Hiraoka K, Shinohara T, Itoh T, Kondo S, Katoh H (2001) RCAS1 as a tumor progression marker: an independent negative prognostic factor in gallbladder cancer. $\mathrm{Br} \mathrm{J}$ Cancer 85:1922-1927

16. Hiraoka K, Hida Y, Miyamoto M, Oshikiri T, Suzuoki M, Nakakubo Y, Shinohara T, Itoh $\mathrm{T}$, Shichinohe $\mathrm{T}$, Kondo $\mathrm{S}$, Kasahara N, Katoh H (2002) High expression of tumor-associated antigen RCAS1 in pancreatic ductal adenocarcinoma is an unfavorable prognostic marker. Int J Cancer 99:418-423

17. Okada T, Iiai T, Kawachi Y, Moroda T, Takii Y, Hatakeyama K, Abo $\mathrm{T}$ (1995) Origin of CD57+ T cells which increase at tumour sites in patients with colorectal cancer. Clin Exp Immunol 102:159-166

18. Mantovani A, Allavena P, Sica A, Balkwill F (2008) Cancerrelated inflammation. Nature 454:436-444

19. Sonoda K, Miyamoto S, Yotsumoto F, Yagi H, Nakashima M, Watanabe T, Nakano H (2007) Clinical significance of RCAS1 as a biomarker of ovarian cancer. Oncol Rep 17:623-628

20. Dutsch-Wicherek M, Wicherek L (2008) The association of RCAS1 serum concentration with the reversibility or irreversibility of the process of immune cytotoxic activity restriction during normal menstrual cycle, cancer relapse, and surgical treatment for various types of squamous cell carcinomas and adenocarcinomas. Am J Reprod Immunol 59:266-275

21. Wicherek Ł, Dutsch M, Mak P, Klimek M, Skladzien J, Dubin A (2003) Comparative analysis of RCAS1 level in neoplasms and placenta. Acta Biochim Pol 50:1187-1194

22. Akashi T, Oimomi H, Nishiyama K, Nakashima M, Arita Y, Sumii T, Kimura T, Ito T, Nawata H, Watanabe T (2003) Expression and diagnostic evaluation of the human tumor-associated antigen RCAS1 in pancreatic cancer. Pancreas 26:49-55

23. Wicherek L (2009) Alterations in RCAS1 serum concentration levels during menstrual cycle in patients with uterine leiomyoma and lack of analogical changes in adenomyosis. Gynecol Obstet Investig 67:195-201

24. Coban S, Ozkan H, Köklü S, Yüksel O, Koçkar MC, Akar T, Ormeci N (2006) The utility of serum receptor-binding cancer antigen expressed on SiSo cells in gastrointestinal tract cancers. Can J Gastroenterol 20:593-596

25. Waldmann TA (1993) The IL-2/IL-2 receptor system: a target for rational immune intervention. Immunol Today 14:264-270

26. Baxevanis CN, Dedoussis GV, Papadopoulos NG, Missitzis I, Stathopoulos GP, Papamichail M (1994) Tumor specific cytolysis by tumor infiltrating lymphocytes in breast cancer. Cancer 74 : 1275-1282

27. Parmiani G, Anichini A, Fossati G (1990) Cellular immune response against autologous human malignant melanoma: are in vitro studies providing a framework for a more effective immunotherapy? J Natl Cancer Inst 82:361-370

28. Parmiani G (1990) An explanation of the variable clinical response to interleukin 2 and LAK cells. Immunol Today 11:113-115

29. Coventry BJ, Weeks SC, Heckford SE, Sykes PJ, Bradley J, Skinner JM (1996) Lack of IL-2 cytokine expression despite Il-2 messenger RNA transcription in tumor-infiltrating lymphocytes in primary human breast carcinoma: selective expression of early activation markers. J Immunol 156:3486-3492

30. Miescher S, Whiteside TL, Moretta L, Von Fliedner V (1987) Clonal and frequency analyses of tumor infiltrating $\mathrm{T}$ lymphocytes from human solid tumors. J Immunol 138:4004-4011

31. Sheu BC, Lin RH, Ho HN, Huang SC (1997) Down-regulation of CD25 expression on the surface of activated tumor-infiltrating lymphocytes in human cervical carcinoma. Hum Immunol 56:39-48

32. Wyckoff J, Wang W, Lin EY, Wang Y, Pixley F, Stanley ER, Graf T, Pollard JW, Segall J, Condeelis J (2004) A paracrine loop between tumor cells and macrophages is required for tumor cell migration in mammary tumors. Cancer Res 64:7022-7029

33. Pollard JW (2004) Tumor-educated macrophages promote tumor progression and metastasis. Nat Rev Cancer 4:71-78

34. Vakkila J, Lotze MT (2004) Inflammation and necrosis promote tumor growth. Nat Rev Immunol 4:641-648

35. Mantovani A, Sozzani S, Locati M, Allavena P, Sica A (2002) Macrophage polarization: tumor-associated macrophages as a paradigm for polarized M2 mononuclear phagocytes. Trends Immunol 23:549-555 
36. Mantovani A, Schioppa T, Porta C, Allavena P, Sica A (2006) Role of tumor-associated macrophages in tumor progression and invasion. Cancer Metastasis Rev 25:315-322

37. Bottazzi B, Polentarutti N, Acero R, Balsari A, Boraschi D, Ghezzi P, Salmona M, Mantovani A (1983) Regulation of the macrophage content of neoplasms by chemoattractans. Science 220:210-212

38. Polverini P, Leibovich SJ (1984) Induction of neovascularization in vivo and endothelial proliferation in vitro by tumor-associated macrophages. Lab Invest 51:635-642

39. Suehiro Y, Muta K, Nakashima M, Abe Y, Shiratsuchi M, Shiokawa S, Ikuyama S, Yoshikawa Y, Watanabe T, Nishimura J (2005) A novel mechanism in suppression of erythropoiesis during inflammation: a crucial role of RCAS1. Eur J Haematol 74:365-373

40. Matsushima T, Nakashima M, Oshima K, Abe Y, Nishimura J, Nawata H, Watanabe T, Muta K (2001) Receptor binding cancer antigen expressed on SiSo cells, a novel regulator of apoptosis of erythroid progenitor cells. Blood 15:313-321

41. Enjoji M (2006) RCAS1-expressing macrophages in inflammatory liver diseases. Liver Int 26:385-387

42. Dutsch-Wicherek M, Tomaszewska R, Stręk P, Wicherek Ł, Składzień J (2006) The analysis of RCAS1 and DFF-45 expression in nasal polyps with respect to immune cells infiltration. BMC Immunol 7:4

43. Dutsch-Wicherek M, Tomaszewska R, Lazar A, Strek P, Wicherek Ł, Piekutowski K, Jóźwicki W (2010) The evaluation of metallothionein expression in nasal polyps with respect to immune cell presence and activity. BMC Immunol 11:10

44. Whiteside TL (2010) Immune responses to malignancies. J Allergy Clin Immunol 125:S272-S283

45. Knutson KL, Disis ML, Salazar LG (2007) CD4 regulatory cells in human cancer pathogenesis. Cancer Immunol Immunother $56: 271-285$ 